\title{
All-Atom Molecular Dynamics Simulations of the Temperature Response of Densely Grafted Polyelectrolyte Brushes: Supporting Information
}

Harnoor Singh Sachar, ${ }^{1}$ Bhargav Sai Chava, ${ }^{1}$ Turash Haque Pial, ${ }^{1}$ and Siddhartha Das ${ }^{1 *}$

${ }^{1}$ Department of Mechanical Engineering, University of Maryland, 4298 Campus Drive, College Park, MD 20742

*Email: $\underline{\text { sidd@ } @ \text { umd.edu }}$ 


\section{S1. Autocorrelation Function for Average End-to-end Brush Height}

In Fig. S1, we plot the autocorrelation function corresponding to the average end-point height of the PE brushes (for various temperatures and grafting densities) over the production run. For a given statistical variable $X$, its autocorrelation function is defined as $C_{X}(t)=\frac{\langle(X(t)-\langle X\rangle)(X(0)-\langle X\rangle)\rangle}{\left\langle X^{2}\right\rangle-\langle X\rangle^{2}}$, where $\langle X\rangle$ represents the mean value of $X$.

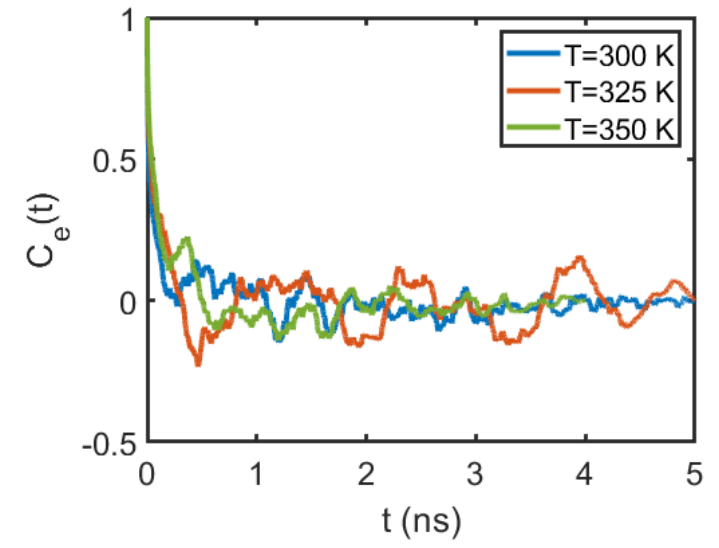

(a)

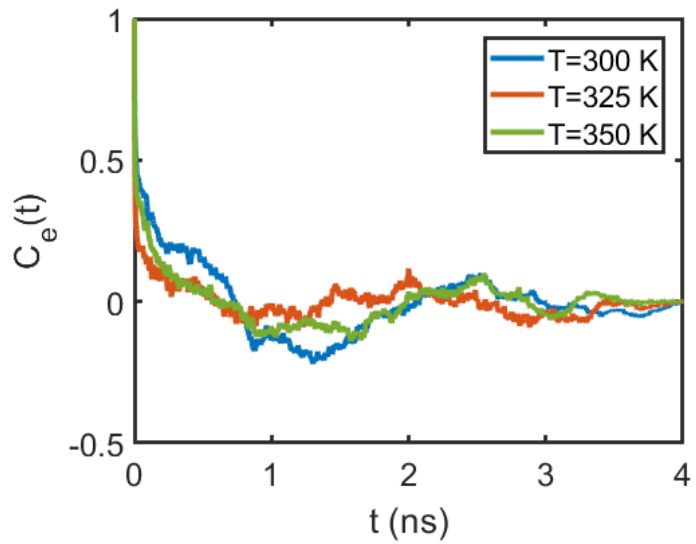

(b)

Figure S1: Autocorrelation function of the average end-point brush height over the production

run for various temperatures. (a) $\sigma_{g}=0.1 / \sigma^{2}$, and (b) $\sigma_{g}=0.2 / \sigma^{2}$.

The average end-point brush height $\left(\left\langle z_{e}\right\rangle\right)$, equilibration time $\left(\mathrm{T}_{\text {equi }}\right)$, and production run time $\left(\mathrm{T}_{\text {prod }}\right)$ for PE brushes at various temperatures and grafting densities are provided in Table $\mathrm{S} 1$. The autocorrelation functions capture multiple large time period oscillations in end-point brush height, which is an indicator that the production run times are adequate to ensure sufficient statistical sampling. Moreover, we observed that our results did not show significant change (both qualitatively and quantitatively) upon increasing the production run from 2 ns to the eventually 
used production runs of 4-5 ns. This too increases our confidence in the computational accuracy of our results.

\begin{tabular}{|c|c|c|c|c|}
\hline $\boldsymbol{\sigma}_{\boldsymbol{g}}\left(\mathbf{1} / \boldsymbol{\sigma}^{\mathbf{2}}\right)$ & $\mathbf{T}(\mathbf{K})$ & $<\boldsymbol{z}_{\boldsymbol{e}}>(\AA)$ & $\mathbf{T}_{\text {equi }}(\mathbf{n s})$ & $\mathbf{T}_{\text {prod }}(\mathbf{n s})$ \\
\hline 0.1 & 300 & 44.90 & 32.6 & 5 \\
\hline 0.1 & 325 & 43.06 & 27 & 5 \\
\hline 0.1 & 350 & 41.92 & 24 & 4 \\
\hline 0.2 & 300 & 55.85 & 12 & 4 \\
\hline 0.2 & 325 & 54.65 & 22 & 4 \\
\hline 0.2 & 350 & 53.80 & 26 & 4 \\
\hline
\end{tabular}

Table S1: Average end-to-end brush height, equilibration time, and production run time for PE brushes at various temperatures and grafting densities. 
S2. Transverse Distribution of the Fraction of Water-Water HBs

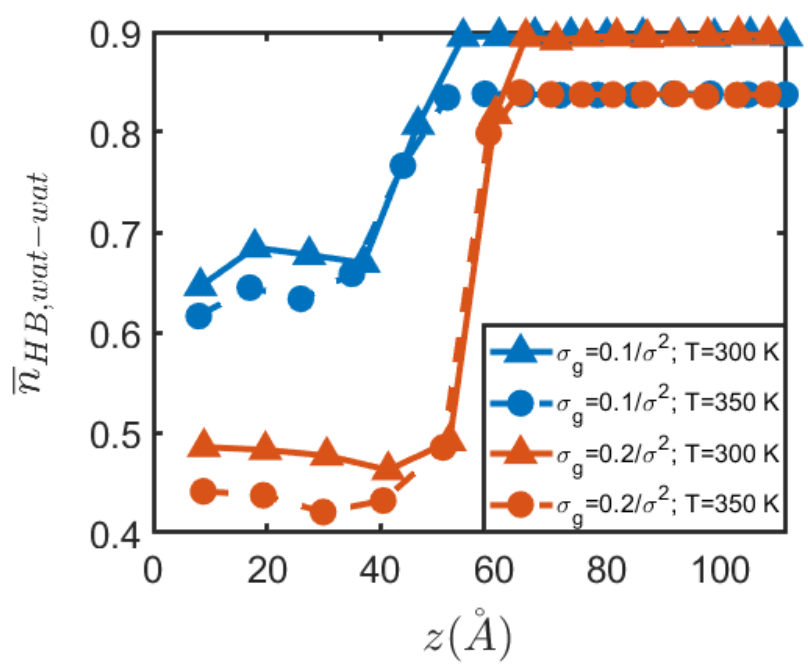

Figure S2: Transverse distribution of the fraction of water-water HBs for various combinations of temperatures and PE brush grafting densities. 
S3. Probability Distribution of the Number of Water-Water HBs Formed by Water Molecules

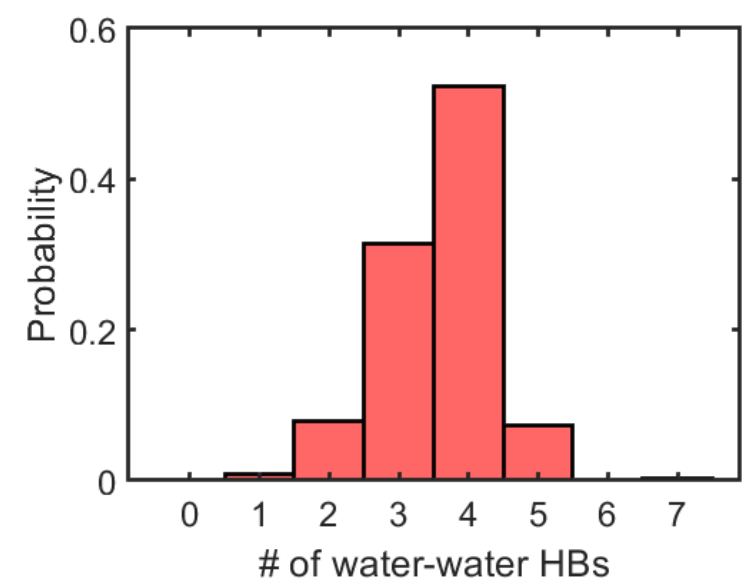

(a)

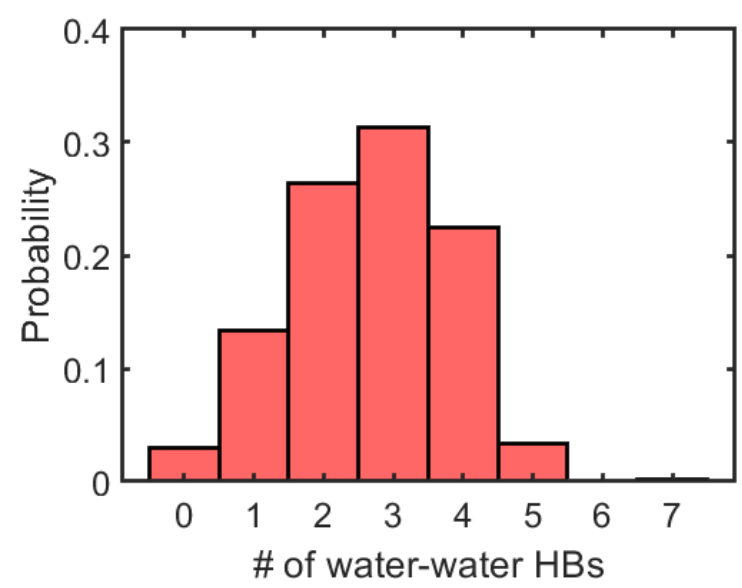

(c)

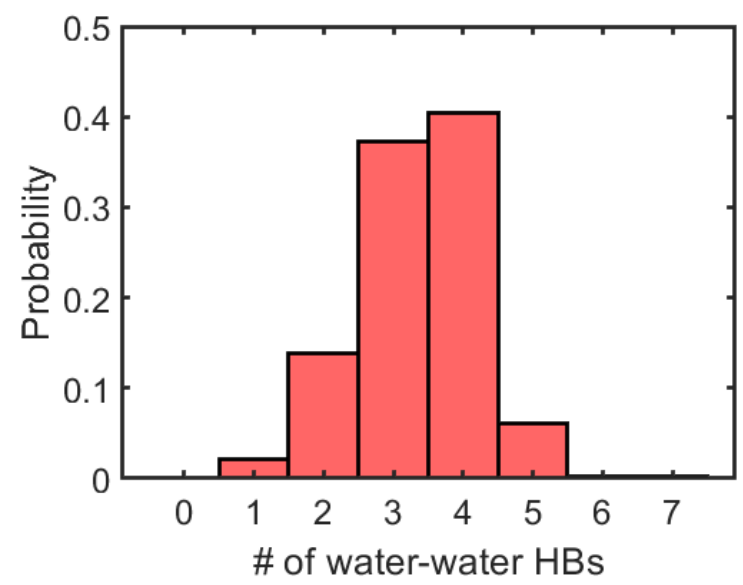

(b)

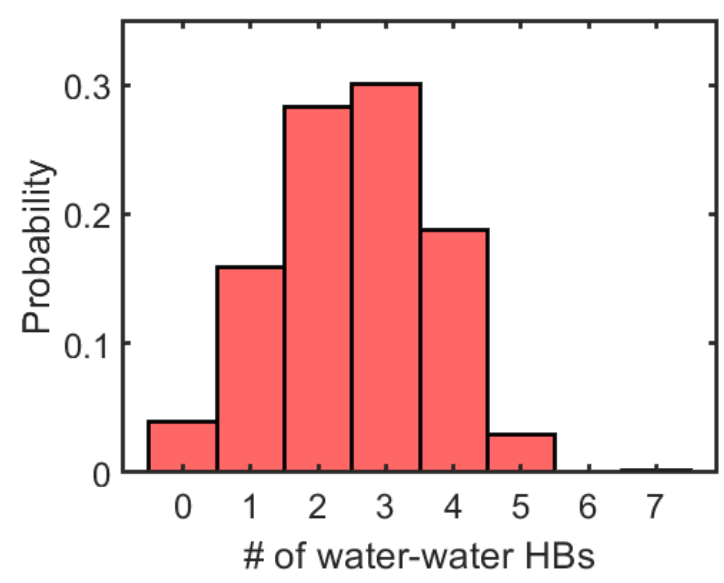

(d) 


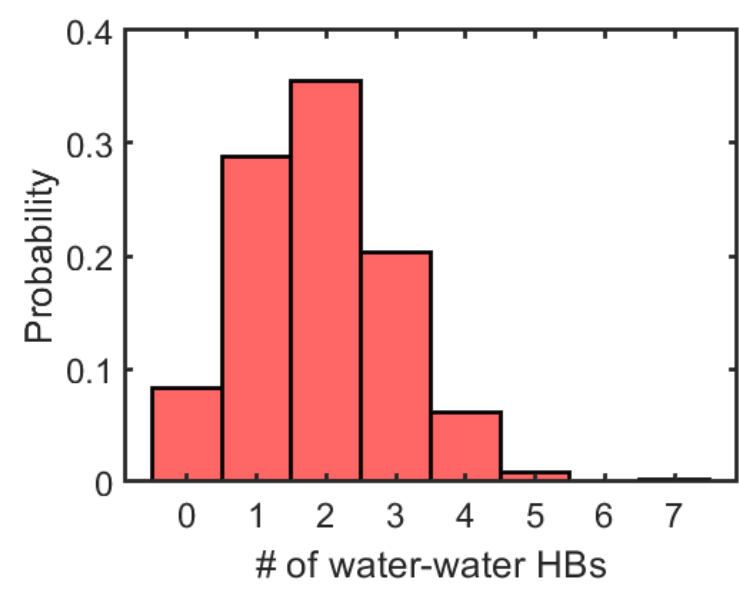

(e)

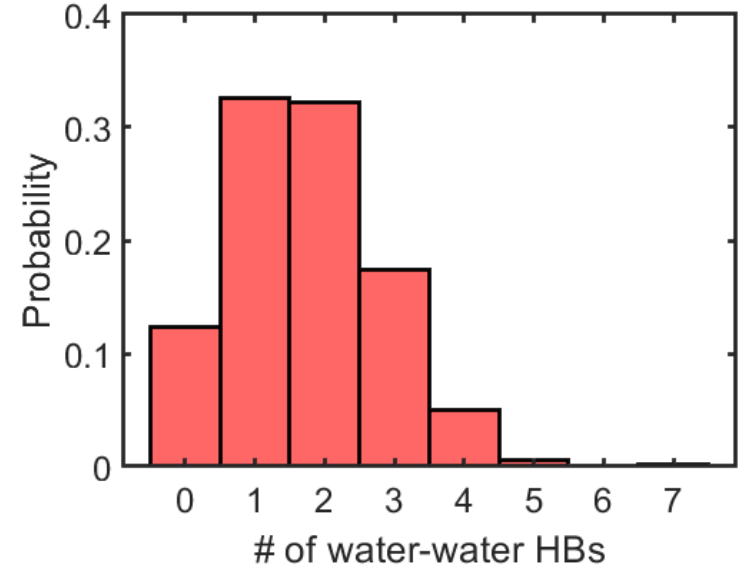

(f)

Figure S3: Probability distribution of the number of HBs formed by water molecules for the

following cases: (a) bulk water at $300 \mathrm{~K}$, (b) bulk water at $350 \mathrm{~K}$, (c) water inside PE brushes with $\sigma_{g}=0.1 / \sigma^{2}$ at $300 \mathrm{~K}$, (d) water inside PE brushes with $\sigma_{g}=0.1 / \sigma^{2}$ at $350 \mathrm{~K}$, (e) water inside PE brushes with $\sigma_{g}=0.2 / \sigma^{2}$ at $300 \mathrm{~K}$, and (f) water inside PE brushes with $\sigma_{g}=0.2 / \sigma^{2}$ at $350 \mathrm{~K}$. 


\section{S4. Mean Squared Displacement of PE Brush-trapped Water Molecules}

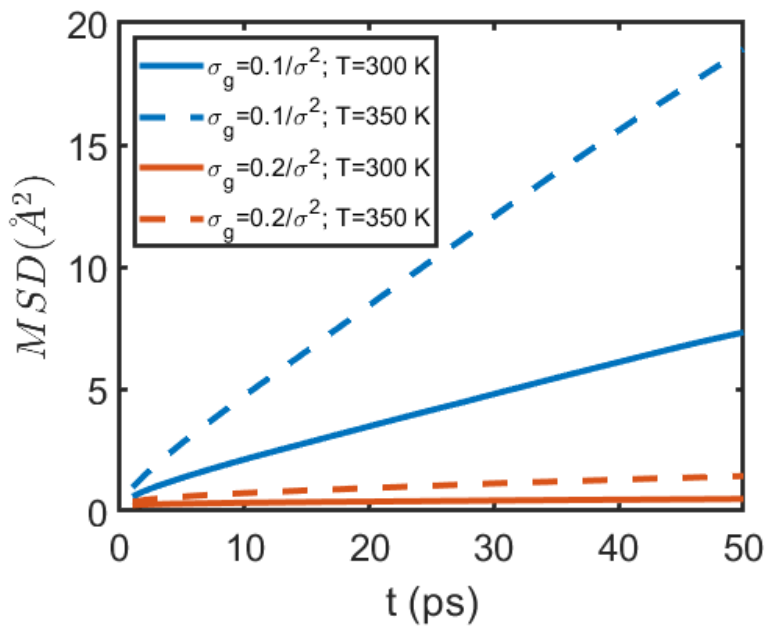

Figure S4: Mean squared displacement (MSD) of water molecules inside the PE brushes for various temperatures and grafting densities. 


\section{S5. Percentage of 'Bound' Water Molecules}

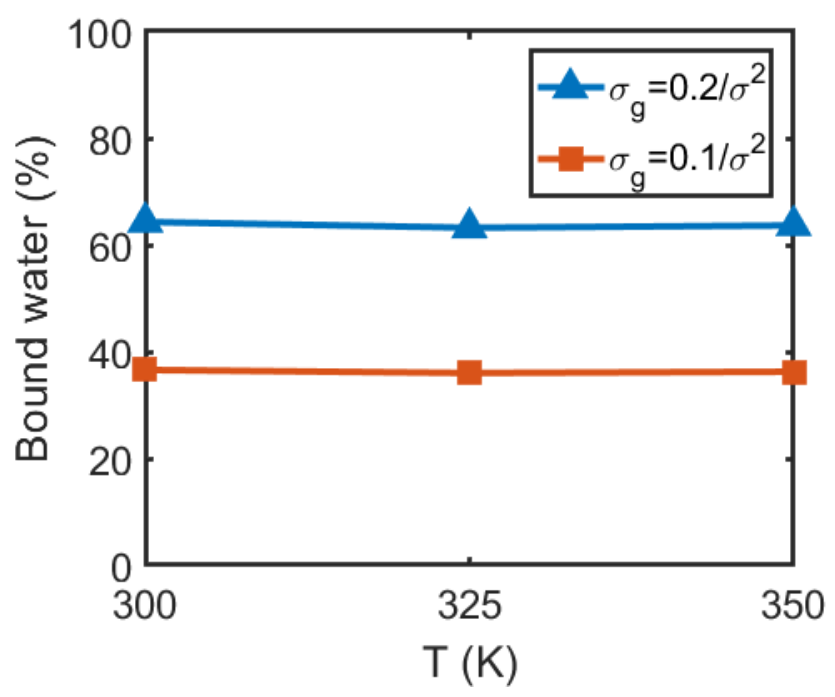

Figure S5: Percentage of 'bound' water molecules inside the grafted PE layer as a function of temperature for various PE brush grafting densities. 


\section{S6. Results for Higher Grafting Density}

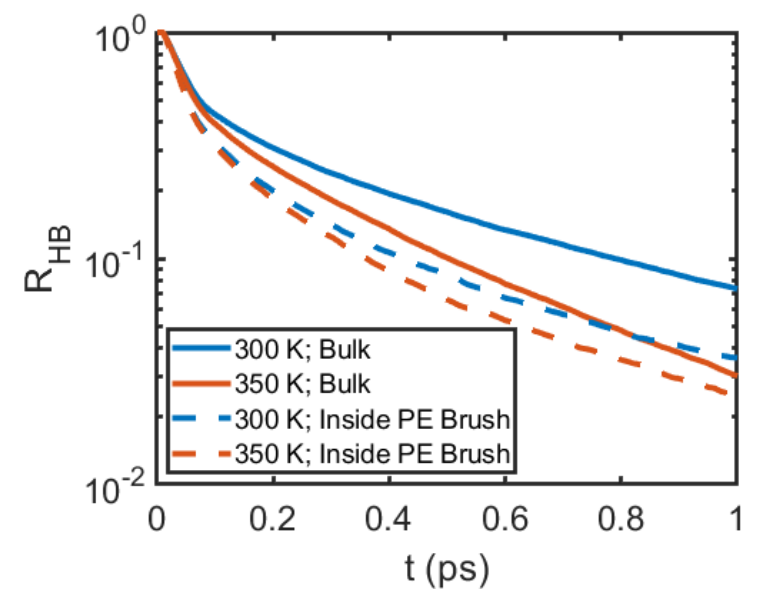

(a)

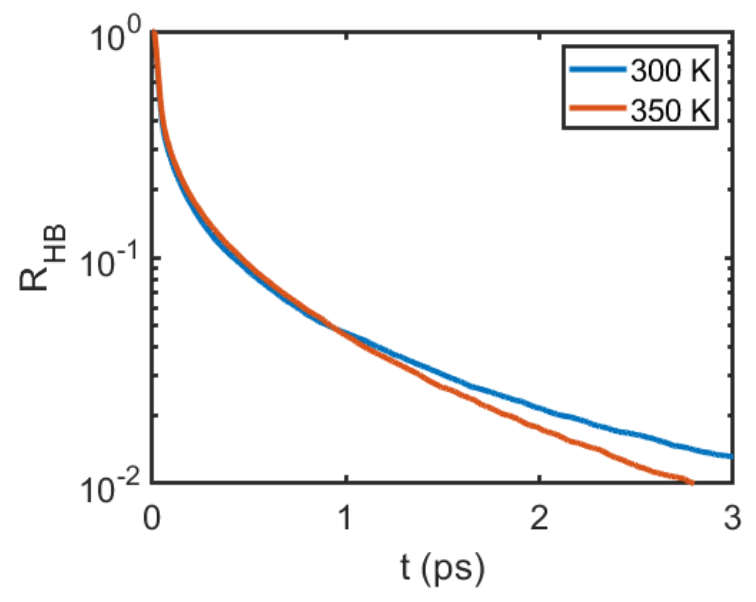

(b)

Figure S6: (a) Residence time distribution for water-water HBs as a function of temperature in bulk water and inside PE brushes with $\sigma_{g}=0.2 / \sigma^{2}$. (b) Residence time distribution for water-PE $H B s$ as a function of temperature inside PE brushes with $\sigma_{g}=0.2 / \sigma^{2}$. 


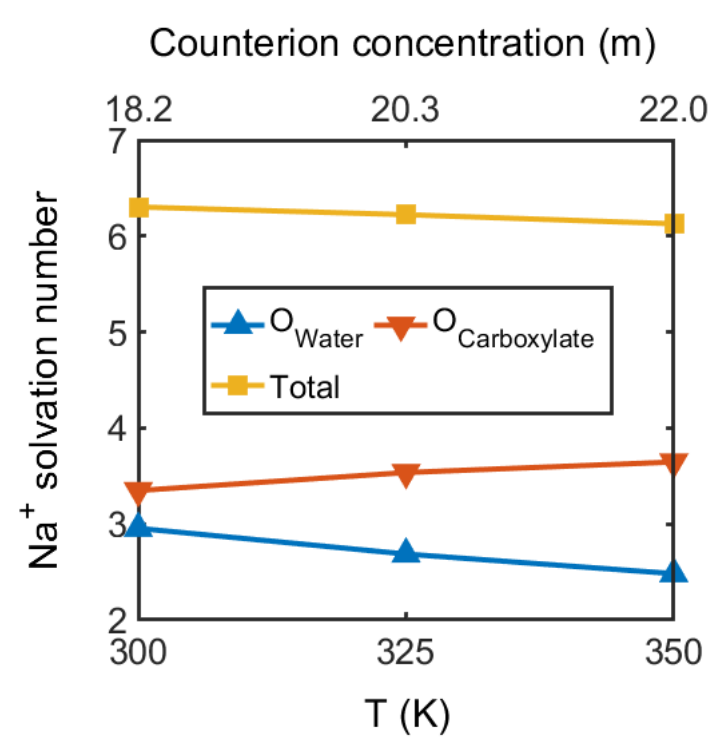

(a)

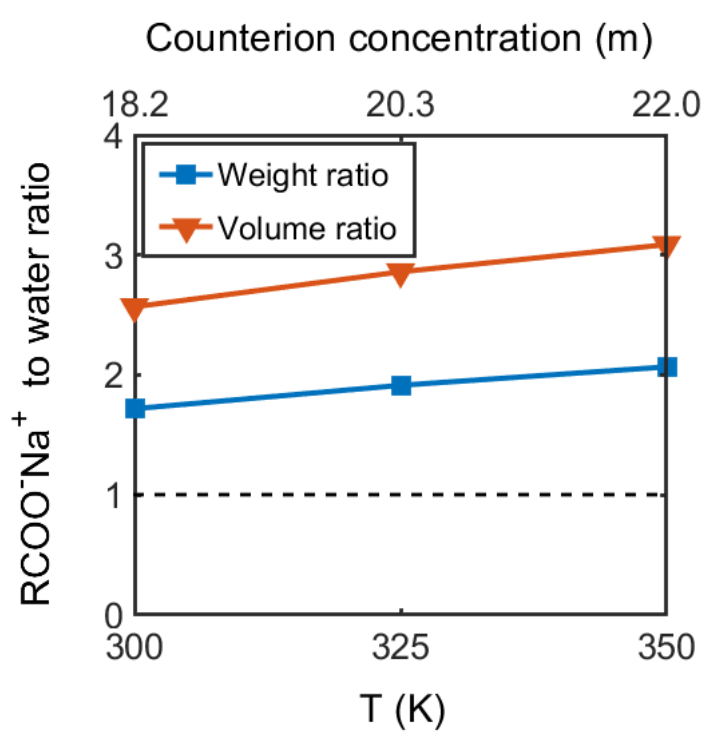

(b)

Figure S7: (a) Variation of the counterion solvation structure inside the grafted PE layer ( $\sigma_{g}=$ $0.2 / \sigma^{2}$ ) with temperature (bottom axis) and counterion molality (top axis). (b) Variation of the 'salt' to water ratio by mass and volume inside the PE brush layer $\left(\sigma_{g}=0.2 / \sigma^{2}\right)$ with temperature (bottom axis) and counterion molality (top axis). 


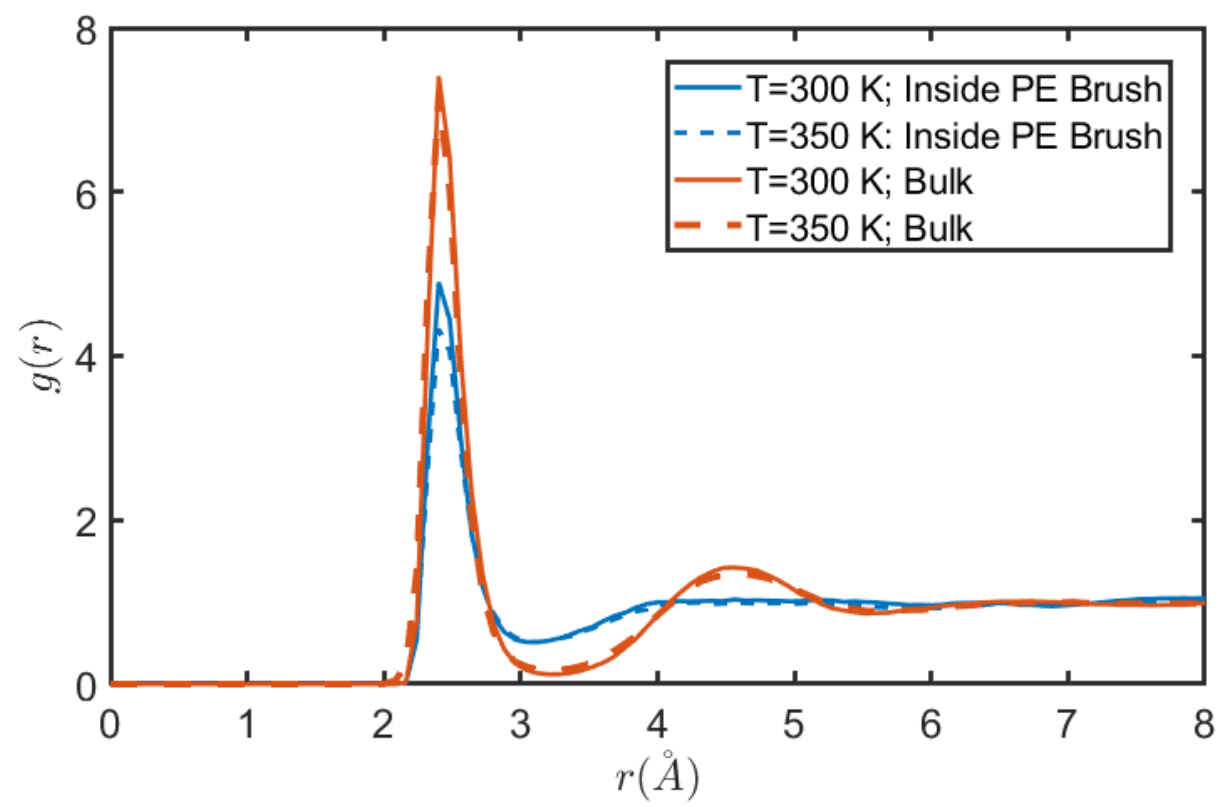

Figure S8: $\mathrm{Na}^{+}-\mathrm{O}_{w} \mathrm{RDF}$ as a function of temperature in 0.1 molal aqueous $\mathrm{NaCl}$ solution and inside the grafted PE layer with $\sigma_{g}=0.2 / \sigma^{2}$. 